zu berücksichtigen. Darüber hinaus erwies es sich als notwendig, einige in den früheren Auflagen etwas stiefmütterlich behandelte Kapitel wesentlich zu erweitern. In erster Linie sind hiervon neben den isocyclischen Verbindungen die heterocyclischen Verbindungen betroffen worden, deren Umfang auf das Doppelte angewachsen ist. Auch das physiologisch-chemische Grenzgebiet ist seiner zunehmenden Wichtigkeit entsprechend etwas stärker als früher berücksichtigt worden. Um das Buch trotzdem nicht ungebührlich anschwellen zu lassen, habe ich vielfach eine etwas gedrängtere Form der Beweisführung gewählt, die, wie ich hoffe, auch in didaktischer Hinsicht eine Verbesserung bedeuten wird. Sodann habe ich manches ausgeschieden, was nicht unbedingt in den Rahmen eines Lehrbuches der organischen Chemie gehört und worüber sich der Studierende bei der weiten Verzweigung unserer Disziplin heute doch zwangsläufig in Speziallehrbüchern orientieren muß. Besondere Mühe habe ich schließlich auch der Revision der physikalischen Konstanten gewidmet, von denen nur die derzeit besten Werte Berücksichtigung gefunden haben.

Berlin, im April 1930.

Friedrich Richter

\title{
Vorwort zur zwanzigsten Auflage
}

Seit dem Erscheinen der 19. Ausgabe ist der Besitzstand der organischen Chemie wieder um zahlreiche Erkenntnisse bereichert worden. Thre theoretischen Grundlagen sind durch physikalische Untersuchungen gefestigt, die Konstitutionsaufklärung wichtiger Naturstoffe und das Verständnis ihrer Rolle im physiologisch-chemischen Geschehen hat erhebliche Fortschritte gemacht. Nicht zuletzt ist schließlich der raschen Umgestaltung auf dem Gebiet der organisch-chemischen Industrie zu gedenken, wo traditionelle Verfahren mit häufig überraschendem Erfolg durch moderne katalytische Methoden verdrängt werden. Die 20. Auflage ist dementsprechend einer sorgfältigen Revision unterzogen und an vielen Stellen neu geschrieben worden. Neu bearbeitet sind unter anderem die Abschnitte über ungesättigte Verbindungen, freie Radikale, Kohlenhydrate, Polysaccharide, alkoholische Gärung, Sterine, Vitamine, Blut- und Blattfarbstoff, Anthocyane und Pyridin. Die eingeschalteten Kapitel über Dipole, Röntgeninterferometrie und thermochemische Messungen werden auch dem Anfänger deutlich machen, welche Verfeinerung unsere Vorstellungen über Bau und Stabilität organischer Verbindungen in neuerer Zeit erfahren haben. Trotz dieses reichlichen Stoffzuwachses ist es auch diesmal wieder durch Verzicht auf didaktisch weniger wichtiges Material gelungen, ohne eine nennenswerte Steigerung des Umfanges auszu. kommen. Es ist mir eine angenehme Pflicht, Herrn Prof. Holleman für mancherlei Anregungen zu danken, die ich bei der Bearbeitung der Neuauflage berücksichtigen konnte.

Berlin, im Juni 1935. 